\title{
BMJ Open A comparison of evidence-based medicine practices between primary care physicians in rural and urban primary care settings in Malaysia: a qualitative study
}

Ranita Hisham, Su May Liew, Chirk Jenn Ng

To cite: Hisham R, Liew SM, $\mathrm{Ng}$ CJ. A comparison of evidence-based medicine practices between primary care physicians in rural and urban primary care settings in Malaysia: a qualitative study. BMJ Open 2018;8:e018933. doi:10.1136/ bmjopen-2017-018933

Received 5 August 2017 Revised 3 May 2018 Accepted 1 June 2018
Check for updates

Department of Primary Care Medicine, University of Malaya, Kuala Lumpur, Malaysia

Correspondence to Assoc. Prof. Dr. Su May Liew; su_mayliew@um.edu.my

\section{ABSTRACT}

Objective This study aimed to compare the evidencebased practices of primary care physicians between those working in rural and in urban primary care settings. Research design Data from two previous qualitative studies, the Front-line Equitable Evidence-based Decision Making in Medicine and Creating, Synthesising and Implementing evidence-based medicine (EBM) in primary care studies, were sorted, arranged, classified and compared with the help of qualitative research software, NVivo V.10. Data categories were interrogated through comparison between and within datasets to identify similarities and differences in rural and urban practices. Themes were then refined by removing or recoding redundant and infrequent nodes into major key themes. Participants There were 55 primary care physicians who participated in 10 focus group discussions $(n=31)$ and 9 individual physician in-depth interviews.

Setting The study was conducted across three primary care settings - an academic primary care practice and both private and public health clinics in rural (Pahang) and urban (Selangor and Kuala Lumpur) settings in Malaysia. Results We identified five major themes that influenced the implementation of EBM according to practice settings, namely, workplace factors, EBM understanding and awareness, work experience and access to specialist placement, availability of resources and patient population. Lack of standardised care is a contributing factor to differences in EBM practice, especially in rural areas. Conclusions There were major differences in the practice of EBM between rural and urban primary care settings. These findings could be used by policy-makers, administrators and the physicians themselves to identify strategies to improve EBM practices that are targeted according to workplace settings.

\section{BACKGROUND}

Evidence-based medicine (EBM), an approach that started in the 1990s, attempts to address the limitations of clinical practices that rely on opinions and the unstructured use of evidence. ${ }^{1}$ Although the concept of EBM has now become widely accepted,
Strengths and limitations of this study

- Findings were analysed from two datasets (rural and urban settings), allowing an in-depth comparison of the evidence-based medicine practices of primary care physicians in both settings.

- Although two different studies were conducted, both used the same research questions, methodological approach and research team.

- The original datasets from rural and urban settings were not merged to preserve data context and integrity.

- A limitation of this study is that private general practitioners in the rural setting were not interviewed, limiting the generalisability of the results.

- The qualitative design does not allow for generalisation of the findings.

issues still remain regarding its adoption in clinical practice. ${ }^{2}$ This challenge is likely to be of particular concern in areas that are resource limited, where factors such as differences in health conditions, availability of resources, lack of good quality research and differences in patient populations have been viewed as limiting the relevance of evidence to clinical practice. ${ }^{3}$ All of these factors indicate that differences exist between the practice of EBM in rural and urban settings; however, studies comparing the two settings are scarce.

Studies in the rural setting have found that the level of evidence-based practice is low due to poor awareness, knowledge gaps, attitudes and lack of resources. ${ }^{45}$ Physicians in urban settings seemed to have better EBM awareness and better availability of resources. Furthermore, workplace factors were found to influence the practice of EBM in urban settings. ${ }^{6}$ The literature has also described differences between rural and urban practice that are likely to affect the practice of EBM 
in these settings such as differences in the availability of continuing professional training. ${ }^{8}$

The aim of this study is to explore the differences in EBM practices in rural and urban primary care settings. This comparison will help to highlight issues relevant to the practice of EBM across different settings.

\section{METHODS}

This is a reanalysis of data from two previous studies in Malaysia: the Front-line Equitable Evidence-based Decision Making in Medicine (FrEEDoM) study, which was conducted in rural primary care settings, and the Creating, Synthesising and Implementing (CSI) study, which was conducted in urban settings. The methods for these studies have been published elsewhere. ${ }^{56}$

\section{Study design}

Both the rural and urban studies used a qualitative study design to explore primary care physicians' personal views and experiences regarding EBM. Senior physicians were interviewed by semistructured individual in-depth interviews (IDIs) to reduce participation inhibition in focus groups, which may arise from a reluctance to discuss issues with junior physicians under their supervision. Medical officers and junior general practitioners (GPs) were grouped according to their clinical settings and interviewed using focus group discussions (FGDs).

Participants in both studies were asked questions based on the topic guide. The topic guide was developed based on the theory of planned behaviour, an extensively used psychological model for understanding human behaviour. ${ }^{9}$ This framework and findings from other studies in the medical literature were used in the formulation of the interview questions. Further details including the topic guides used can be obtained from the initial two articles. ${ }^{56}$ The interviews and FGDs in the two studies were audio recorded. Field notes were made during the interviews. The duration of the IDIs and FGDs was $45-60$ min per session.

\section{Participant recruitment}

This research started with the FrEEDoM study in which interviews were conducted at rural primary care clinics in the state of Pahang, Malaysia. Four FGDs with 15 medical officers and 3 IDIs with family medicine specialists were carried out. After completion of the rural study, we continued with the CSI study in which interviews were conducted at urban primary care settings in two states: Kuala Lumpur and Selangor, Malaysia. All 39 physicians working in public and private healthcare clinics were recruited purposively through email and telephone invitations.

In total, there were 55 physicians from various backgrounds and settings who participated in this study. The locations and timing of the interviews were at the physicians' convenience. Recruitment was continued until data saturation was achieved. When no new codes or themes emerged from the analysis, this indicated that data saturation was reached. ${ }^{10}$ Data were analysed while data collection was ongoing.

\section{Sampling techniques}

Primary care physicians in rural and urban areas were recruited through purposive sampling. Medical officers and family medicine specialists from public health clinics in urban and rural areas and from private health clinics and tertiary clinics in urban areas were interviewed to achieve maximum variation based on EBM knowledge, working experiences and settings.

\section{Data management}

Data from the two previous qualitative studies were sorted, arranged, classified and compared with the help of qualitative research software (NVivo V.10). Both datasets (data from the FrEEDoM and CSI studies) were kept open and worked with simultaneously. Thematic analysis was used in analysing the data. Data categories were interrogated through comparison between and within datasets to identify similarities and differences in rural and urban practices. The main focus was to highlight the core differences, and therefore, the differences were identified and analysed in a new dataset. The original datasets from the rural and urban settings were not merged to preserve data context and integrity. Only nodes and themes found to be different between datasets were chosen and renamed. This process was done by HR.

Once completed, the similarities and differences were presented to the team (SML and CJN) for review. Themes were then refined by removing or recoding redundant and infrequent nodes. Review of the themes was conducted by returning to the data repeatedly to check for fit. Quotes were annotated by the participant number, gender, position and their location (rural or urban).

\section{Patient and public involvement}

This was a study to compare physicians' views on EBM in different work settings. Public involvement was limited to having non-healthcare personnel involved in the design and conduct of the study. Patients were not involved.

\section{RESULTS}

The demographic and clinical backgrounds of the participants are presented in table 1. Five major themes that had influenced the implementation of EBM in practice emerged from comparison of the datasets: workplace factors, EBM understanding and awareness, work experience and access to specialist placement, availability of resources and patient population. The core differences in the rural and urban primary care settings are shown in box 1 .

\section{Workplace factors}

Important differences in EBM practice existed between settings; this finding was seen between rural and urban workplaces, and among the types of primary care practices, 
Table 1 Demographic and clinical background of participants

\begin{tabular}{|c|c|c|c|}
\hline Characteristics & $\begin{array}{l}\text { No of participants } \\
(n=55)\end{array}$ & $\begin{array}{l}\text { Rural } \\
(n=18)\end{array}$ & $\begin{array}{l}\text { Urban } \\
(n=37)\end{array}$ \\
\hline Age (years) & $28-65$ & $28-48$ & $26-65$ \\
\hline \multicolumn{4}{|l|}{ Gender } \\
\hline Male & 17 & 6 & 11 \\
\hline Female & 38 & 12 & 26 \\
\hline \multicolumn{4}{|l|}{ Position } \\
\hline $\begin{array}{l}\text { Family medicine } \\
\text { specialists (FMSs) }\end{array}$ & 10 & 3 & 7 \\
\hline $\begin{array}{l}\text { Medical officers } \\
\text { (MOs) }\end{array}$ & 37 & 15 & 22 \\
\hline $\begin{array}{l}\text { General } \\
\text { practitioners (GPs) }\end{array}$ & 8 & NA & 8 \\
\hline \multicolumn{4}{|l|}{ Workplace } \\
\hline $\begin{array}{l}\text { Academic primary } \\
\text { care settings }\end{array}$ & 15 & NA & 15 \\
\hline Public health clinics & 32 & 18 & 14 \\
\hline $\begin{array}{l}\text { Private health } \\
\text { clinics }\end{array}$ & 8 & NA & 8 \\
\hline $\begin{array}{l}\text { Years of practice } \\
\text { (years) }\end{array}$ & $3-36$ & $3-28$ & $1-36$ \\
\hline
\end{tabular}

Ever attended an EBM training course

\begin{tabular}{llll} 
Yes & 14 & 2 & 12 \\
No & 41 & 16 & 25 \\
\hline
\end{tabular}

FMS=physicians with postgraduate qualifications practising in public health clinics.

MOs=physicians without postgraduate qualifications practising in public health clinics.

$\mathrm{GPs}=$ physicians practising at private clinics.

EBM, evidence-based medicine; NA, not applicable.

that is, academic, private and public primary care settings. Participants noted that a reason for these disparities was the difference in the physician-to-patient ratio. All public health clinics had to manage large numbers of patients; however, in rural clinics, this problem was compounded by a lack of physicians, especially in small clinics serving isolated areas. Therefore, those practising in rural areas felt that it was not feasible to practise EBM.

In rural areas, there is one physician to so many thousand patients, so it depends on how fast they can see

Box 1 Major differences between rural and urban primary care settings

Rural and urban divide.

Workplace factors.

- Physicians' understanding and awareness of evidence-based medicine (EBM).

- Work experience and access to specialist placement.

- Accessibility and availability of resources.

- Patients' acceptance towards EBM. their patients again. They might see them one month later, but for us in urban areas, it's very easy; we retain the folder and the patient can come the next day, we can see them within a week. (P26, medical officer, academic primary care, urban)

Health system practices also differed between the rural and urban practices, which could also affect the practice of EBM. A participant gave an example of postnatal care where nurses can still manage to have a scheduled visit in rural practices even though the population is large and scattered.

From what I heard, for example, postnatal care, the staff nurse (especially the one in my healthcare clinic) and also in Pahang, they are really concerned about their patients, visiting them according to the days. But, in Selangor (a state in Malaysia), I heard that even at one day postnatal, a staff nurse who comes to the house is like something huge because they got too many patients. They can't, so even though Pahang is a big area, I guess the population is scarce, so they can still do this. So, I feel that sometimes the research conducted in urban areas can't be applied here. (P12, medical officer, primary care clinic, rural)

Another participant agreed that the situations at the different settings are not the same.

It's not the same, different settings are different. I find the KKM [Ministry of Health] recommendations are a bit different from what we do here. Here, [academic primary care], our environment encourages evidence-based medicine. But, in KKM, it's mostly just based on expert opinion. Whatever your boss says, you just follow, no questions! (P14, medical officer, academic primary care, urban)

Lack of standardisation in care also contributed to differences in EBM practices. This was especially marked in rural areas where limited resources such as hospital beds and medications meant that physicians had to adhere to the known practices of the specialist in charge of the district. For example, in the state of Pahang, a system known as the 'buddy system' was established. Every primary care physicians in a district was assigned his or her own 'buddy', a specialist in obstetrics and gynaecology (O\&G) working in the closest referral hospital. This arrangement was found to be useful because the physicians felt that they could rely on their 'buddy' to get answers.

And luckily, we have our buddy! In Pahang, we have a buddy, a buddy system. For O\&G, they give one O\&G specialist to cover this area, so we can contact our buddy. Quite easy to get answers because sometimes we don't have enough time to go to the computer and check for the evidence-based answers; we just call the O\&G specialist and straight away we get the right answer (giggle). I'm not sure whether this is only in Pahang or not. Only O\&G. I find it helpful because 
we know our buddy and our buddy also knows us, and two-way communication is better because sometimes, if you don't have the buddy, you will call the 'on call' people and it's quite disturbing. (P1, family medicine specialist, primary care clinic, rural)

However, the lack of standardisation in clinical practice meant that rural physicians needed to know and follow the individual clinical practices of different specialists. If a patient required referral to secondary care, the primary care physician would have to decide where to refer to and manage the patient according to the practices of the specialist in charge at that hospital.

\section{Physicians' understanding and awareness of EBM}

Differences and similarities in the understanding and awareness of EBM among physicians emerged during data analysis. There was almost no EBM practice in the rural setting except by family medicine specialists. Both rural and urban physicians viewed EBM as being restricted to guidelines, research and statistics.

I think it's because they don't know what to do. If you know what to do, usually you won't spend longer time. Like I said, you search, you just blindly search like that and then you end up with all these things [results], right. At the end of the day, you are going to give up! But if you know the technique, what's the best... then you just go for it, but some don't know how to search. (P5, family medicine specialist, primary care clinic, rural).

Urban physicians in this study appeared to be more aware about EBM.

Evidence-based medicine is actually done based on their study research. So, most of it is based on randomized controlled trials. So, I think it is very accurate in terms of managing patients; this is best for the patients because they have studied and did research on it. So, that's why they have come out with something and we try to apply it on our patients. (P23, medical officer, academic primary care, urban).

\section{Work experience and access to specialist placement}

One aspect that emerged from the data in this study was that the rural physicians were mainly junior physicians with less work experience who were posted there as part of the mandatory government service. The junior rural physicians voiced uncertainty regarding clinical practice and did not know how to search for information. The only reliable source of information was hospital specialists who were sometimes difficult to contact.

When we are really not sure, we hope that they can give us answers, but sometimes it's just difficult and we have to consult another doctor. It's frustrating! It happened many times, the MO (medical officer) cannot decide; then, they ask to call their specialist. When you call, sometimes they are at places with no coverage, then you have to try a few times, so it's difficult! Even though the MO is a very good senior. (P21, medical officer, primary care clinic, rural)

As specialists were difficult to contact, social apps such as WhatsApp were highlighted by many of the rural junior physicians as constituting an important medium of information. This app allowed them to source answers to clinical questions from their peers-a social group that can be contacted easily and quickly.

It's our friends. Actually, it's the fastest. For example, during an emergency setting and when we want an answer, that's the fastest medium! I mean, of course you can run home, Google and read one-by-one, but that's not practical! (P8, medical officer, primary care clinic, rural)

The junior rural physicians in this study viewed searching for answers on the internet as being impractical due to the time factor and lack of facilities. In comparison, the practice of searching for answers in urban settings was different because of easier accessibility to information sources. Physicians in urban practices in this study were older and preferred using direct communication rather than using mobile apps.

Hmm, I don't have WhatsApp or a smartphone, so I just call someone up. (GP31, general practitioner, urban)

Some of the senior physicians were worried about the gap between the junior and senior primary care physicians. There has been a dramatic increase in the number of medical universities and an increase in junior physicians being posted to public health clinics. The quality of the junior physicians has been a substantial concern to the senior primary care physicians.

Maybe they can sort of make it as an arahan [an order] for all the physicians; in fact, our Health Department is actually worried because we are getting junior physicians and medical officers, and now they are getting housemen in clinics. We are so worried about the quality of the physicians, whether they are actually as good as previous physicians, you know, like before. Not sure whether these junior physicians are exposed to EBM or not because so many of them, we don't know what they have been taught. So, the Director can set the rules for all physicians, especially the junior ones, to be exposed to EBM. At the end of the day, all we are talking about is the benefit of the patient. (P32, general practitioner, private health clinic, urban)

Senior physicians also reported that the practice of sending new junior physicians to rural clinics would create problems as there is a lack of experienced physicians in those locations.

I think because in Hospital X [hospital in a larger district in Pahang] now, most of them are new 
physicians; when you work as a houseman in Kuala Lumpur (national capital of Malaysia) and after finishing housemanship, you become a MO, you have to go up to the rural area or somewhere else, right? Sometimes when we call them, they are not sure, and they ask us to call their specialist! It makes our life quite difficult too because we have to call the physicians and recite the history again and again, right? I understand, but I feel that they just don't have the confidence ... (laugh) (P1, family medicine specialist, public health clinic, urban)

\section{Accessibility and availability of resources}

Although resources were limited for both settings, the effects were more impactful for rural practices. Restrictions on the accessibility and availability of resources were highlighted to be a major issue influencing EBM practice.

We have some medications here, which I do try to limit, and we are not able to freely purchase any drugs here, it has to be under the ' $\mathrm{A}$ ' list. If you have a beta blocker, I would have only two or three types of beta blockers in that drug group, whereas the hospital would have so many types. (P19, medical officer, primary care clinic, urban)

It was felt that the availability of many different types of medications and facilities was crucial to the practice of EBM and that this circumstance was one of the reasons why it was harder to practise EBM in rural settings. There were participants who felt that they could not practise EBM at times because of the limited selection of medications and that decisions were made simply on the basis of what was available.

But usually we won't be able to practice based on evidence because we have very limited resources back in clinic. Yes, based on evidence of course, the best medication would be this, this, this and yet we are limited to this kind of, we only have this; by hook or by crook, you have to manage your patient with the resources that you have. We want to go a step ahead. For example, in terms of anti-hypertensives, beta-blockers, nifedipine, we still give nifedipine in practice, amlodipine, and some of them, they cannot tolerate this, and you know we need some ARBs or whatever but we can't go the extra mile to have ... (P10, medical officer, primary care clinic, rural)

Even when the doctor knew about studies showing that certain drugs were harmful, he/she had to continue practising the old methods, which are non-evidence based, due to the lack of resources.

It's just that the access that we have is very limited for us; I think that is what our bosses need to consider because it's all not evidence-based medicine and we've been practising the old methods. Even in terms of bringing down the $\mathrm{BP}$ in a hypertensive emergency, and now we are not supposed to be practising using nifedipine because there are studies saying that it will cause hypoperfusion. But, we are still using nifedipine as for now, and it's not our own initiative to go and do research ourselves. (P10, medical officer, primary care clinic, rural)

Restrictions to resources affecting EBM practice include accessibility to secondary care. Physicians had to consider hospital access for patients requiring secondary care in terms of distance and availability.

Yes, it's difficult! It depends on medication-wise, the availability of medications and services. For example, a pregnant diabetic patient, by evidence, we have to refer for an eye check-up referral and all, but when you are in a rural setting, you have to take into account for the patient to go there [clinic], so if they can go, we will refer, and then, the waiting time. It's not like we cannot proceed based on the clinical guidelines, it's the easy access for that that counts. (P5, family medicine specialist, primary care clinic, rural)

Geographical distance to medical care is a problem for rural practices, and this issue did not emerge in the interviews with physicians practising in urban settings. Physicians found it difficult to help patients. They were concerned that patients would struggle to travel to the hospital and that they may not be able to afford the cost of travelling and hospitalisation.

We just tell our patients, 'we are sorry, this is the only thing that we have in the clinic' and if you want to go further, then just write a letter for them to be referred, but some of them they couldn't, those in this kampung [village] setting, as you said, in a rural setting, right? Travelling to other centres, like going all the way to Temerloh (district in Pahang) for follow up, it's really a huge deal for them because it's not like us earning monthly; they go there, they leave their family behind, they don't get paid for that particular day, so it's really a struggle for them. (P10, medical officer, primary care clinic, rural)

The greater distances found in rural settings also meant that access to training for the rural physicians was difficult. A participant suggested that teleconferencing could be useful to overcome this problem.

It's different! Rural areas, they have one clinic, one or two MOs only. The distance is like one to two hours from the main clinic. Rural areas, I suggest doing video trainings. I think it is good, also because that's what I did when I did my rural posting attachment in Canada. That area was quite rural, and it was like a rural family medicine practice, the family doctor would be in a room with a computer, and CME is conducted from the hospital in Calgary for, like, four, five hours once a week. (P6, family medicine specialist, primary care clinic, rural) 


\section{Patients' acceptance of EBM}

Patients were considered an important factor influencing the physicians' practice of EBM. This finding was observed for both rural and urban physicians. However, rural patients prefer traditional medicine.

Because that day we conducted research in a rural area, they have the Orang Asli [aborigines], their beliefs and things like that, so many traditional herbal medicines and all, you cannot change their practice. (P26, medical officer, tertiary hospital, urban)

Bomoh! (Shaman!) Normally they believe the Bomoh (Shaman) rather than a doctor because they are more human. Yes! It happens in urban areas too! Not only in rural areas. (P27, medical officer, tertiary hospital, urban)

A lot, we have a lot of patients who are on alternative complementary treatment. All the herbs and traditional medicines. (P21, medical officer, public primary care, rural)

Urban patients were viewed as being more knowledgeable with some doing their own health research before consulting the doctor.

See, we live in a population where they are all retired professionals. They read, and they are proficient in English, so they understand internet English. They Google a lot and they ask for a lot of opinions from people of different backgrounds, then they formulate this kind of opinion for themselves. They self-treat a lot and change their own medication and come up with Google's internet papers. (P31, medical officer, primary care clinic, urban).

\section{DISCUSSION}

The research identified major differences and gaps in the description of EBM practices between rural and urban primary care. Major themes that emerged to indicate differences in the practice of EBM were workplace factors, lack of understanding and awareness of EBM, work experiences and access to specialist placement, accessibility and availability of resources and patients' acceptance of EBM.

The original aim of the research team was to explore the influence of the work setting on EBM practices because previous literature had indicated that the setting could influence EBM practices. We also postulated that physicians in rural settings have limited access to evidence compared with those in urban settings. However, there was a scarcity of research in this area, specifically of practices in the rural setting. Thus, we first explored the research question in the rural setting by the FrEEDoM study ${ }^{5}$ and subsequently in the urban setting by the CSI study. ${ }^{6}$ In-depth exploration of practices in both settings was possible through the use of a qualitative study design, although with the understanding that the findings were not generalisable. The data were subsequently compared with explore differences between the two settings. These findings will also be used to inform the development of an instrument for a quantitative study.

We found differences in the management of healthcare processes between the rural and urban areas, which led to differences in workplace culture towards EBM. These disparities included differing ways in the management of large numbers of patients, in organisational support and in access to specialist care and hospital beds. Interestingly, although the physicians practising in rural settings lauded the 'buddy system', this approach meant that clinical practice was not influenced as much by evidence as towards expert opinion. Patient management differed according to the specialist managing the case. This situation was not found in the urban setting, where accessibility towards tertiary care was easier. Our findings indicated that there was a variation in practice that was influenced by expert opinion rather than by the use of research evidence. Some variation is useful as this indicates consideration of individual preferences; however, too much variation leads to inequity in healthcare, meaning that some patients may not receive the best of care simply because of geographical location. ${ }^{11}$

We also found that awareness regarding EBM was poorer in the rural setting. This finding was similar to the results of other studies, ${ }^{12}{ }^{13}$ which also found that primary care physicians practising in rural settings lacked awareness regarding EBM. A contributing factor may be the lack of accessibility to EBM training in rural settings. It is difficult to attend conferences and workshops in rural settings due to the remoteness of the locations. The limited numbers of physicians in rural clinics also meant that physicians needed to take turns to attend these courses. Urban practice offered a greater opportunity to attend training, especially for those whose practices were located at academic hospitals.

Our study further highlighted the difficulties that arise from remote rural postings. Within the Malaysian health system, junior physicians are more likely to be sent to rural postings compared with experienced physicians. Junior physicians are retained in public services through a 3-year compulsory service scheme. Furthermore, rural postings are often regarded as a necessary requirement for applying for specialty training ${ }^{14}$ Thus, junior physicians posted in rural areas often lack insight and experience in the management of diseases. ${ }^{14} 15$ This factor is likely to affect the quality of healthcare services in rural areas. The EBM approach was considered to be an approach that could help the physicians make better decisions in rural settings. ${ }^{6}$ However, this promise remained unfulfilled because the junior physicians lacked understanding of EBM and skills in its practice.

Similar to other studies, ${ }^{16-18}$ the availability and accessibility of resources including drugs and facilities were perceived as a major barrier to the practice of EBM. However, in this study, these circumstances also appeared to affect the urban setting, possibly because resources in 
a low/middle-income country are limited, even in urban areas. However, this study did find that this issue was of greater importance in the rural areas where the lack of resources was greater compared with urban settings. For the practice of EBM to improve, it is not enough to simply increase resources for the health clinics-a wider perspective is needed. Our findings have shown that rural postings must be made more engaging to ensure an adequate supply of primary care physicians. The current incentive plans to retain experienced physicians in rural settings are inadequate. ${ }^{19}$ In addition, non-financial incentives could be made available such as a reduction in work hours and the availability of good schools and other resources important to retain physicians in rural areas. ${ }^{20-23}$

Finally, there were perceived differences in the patients' acceptance of EBM. In rural areas, traditional medicine and other complementary medicine practices appeared to be highly regarded and practised compared with those practices and perceptions among urban populations. In the urban areas, patients' acceptance of EBM was also challenging due to the population having assumptions and expectations from various information sources. They also tended to be more assertive at times with their decisions. This led to feelings of frustration among the physicians who felt that such attitudes and behaviours hindered the practice of EBM. However, EBM is defined as the integration of research evidence, clinical expertise and patients' values. Patient values and preferences are considered to constitute an important and integral part of this approach. This is where shared decision-making should be incorporated into the consultation process.

The findings from this study are specific to the Malaysian context. There is a lack of literature that compares rural and urban primary care settings. However, there are published studies that have focused only on a particular setting, either rural or urban, and for these studies, there are some similarities to our findings. The similarities found in other rural studies indicate that there is a lack of access to resources, lack of knowledge and skills regarding EBM, high workload, greater time pressure and lack of support from the workplace organisation. ${ }^{24-26}$ Studies conducted in urban settings reported that primary care physicians seemed to have awareness of EBM and that attitudes differed based on work experience. ${ }^{27} 28$

The participants were recruited from rural clinics in Pahang and urban clinics in the Klang Valley. This factor limits generalisability to primary care physicians from other states or locations. However, study participants shared and compared their work experiences in other states and locations during the interviews. Therefore, a wide range of views and important insights were obtained.

Although there were two datasets, the studies were similarly designed to allow for comparison. This new comparison raises the question of whether the questions posed by the interviewers were adequate to answer a different research question (comparison of the urban and rural settings). As this was planned from the start of the research, the topic guide directly addressed the influence of the work setting towards the practice of EBM, which allowed us to answer the research question of this study.

\section{Strengths and limitations}

The strength of this study was that the two studies were conducted separately in two different settings, that is, rural and urban areas, but using the same research question, the same methodological approach and the same research team. The aim of the research team was to explore in-depth views and experiences of primary care physicians working in the different settings. The separate datasets were used to explore similarities and differences. The limitation of this study is that the private GPs in rural practices were not interviewed in the FrEEDoM study, which limits the data analysis process in this study. Finally, the qualitative design does not allow for generalisation of the findings.

However, our aim was to generate understanding of the influences that affect the practice of EBM in the rural and urban settings. Further studies are needed to identify and quantify the actual associated factors.

\section{CONCLUSION AND RECOMMENDATIONS}

There were major differences in the practice of EBM between rural and urban settings, which impacted clinical practice. Workplace factors, healthcare organisation, availability of resources and patient populations were found to differ significantly. Strategies to overcome these issues are required before improvement in the practice of EBM can be seen. It is important that the policy-makers consider changing the system to encourage the practice of EBM in all workplace settings, whether rural or urban. Therefore, it should be ensured that there is equitable and sufficient distribution of resources needed for clinical practice to be evidence based in all primary care settings.

Contributors HR wrote the first draft of the paper and led the revision of the paper, data analysis and interpretation of the data. SML and CJN were involved in the conception, study design, interpretation of the data and review of the paper. All authors read and approved the final manuscript.

Funding The FrEEDoM study was funded by University of Malaya Research Grant (UMRG RP037C-15HTM) and the CSI study was supported by a High Impact Research (HIR) Grant, University of Malaya under grant number (E000078).

Disclaimer The funders had no role in the study design, data collection and analysis, decision to publish or preparation of the manuscript.

Competing interests None declared.

Patient consent Obtained.

Ethics approval Ethics approval for the studies was granted by the National Medical Research Register-Medical Research Ethics Committee (MREC reference: NMRR ID NMRR-12-1262-14539 S2 R0) for the FrEEDoM study and for the CSI study from the University of Malaya Medical Centre Medical Ethics Committee (MREC: 962.9).

Provenance and peer review Not commissioned; externally peer reviewed. Data sharing statement No additional data are available.

Open access This is an open access article distributed in accordance with the Creative Commons Attribution Non Commercial (CC BY-NC 4.0) license, which permits others to distribute, remix, adapt, build upon this work non-commercially, and license their derivative works on different terms, provided the original work is 
properly cited and the use is non-commercial. See: http://creativecommons.org/ licenses/by-nc/4.0/

(C) Article author(s) (or their employer(s) unless otherwise stated in the text of the article) 2018. All rights reserved. No commercial use is permitted unless otherwise expressly granted.

\section{REFERENCES}

1. Kamath S, Guyatt G. Importance of evidence-based medicine on research and practice. Indian J Anaesth 2016;60:622-5.

2. Sur RL, Dahm P. History of evidence-based medicine. Indian Journal of Urology : IJU. Indian Journal of Anaesthesia 2011;24:487-9.

3. Chinnock P, Siegfried N, Clarke M. Is evidence-based medicine relevant to the developing world?: Systematic reviews have yet to achieve their potential as a resource for practitioners in developing countries. Evid Based Complement Alternat Med 2005;2:321-4.

4. Wakerman J, Humphreys JS, Wells R, et al. Primary health care delivery models in rural and remote Australia: a systematic review. BMC Health Serv Res 2008;8:276.

5. Hisham R, Liew SM, Ng CJ, et al. Rural Doctors' Views on and Experiences with Evidence-Based Medicine: The FrEEDoM Qualitative Study. PLoS One 2016;11:e0152649.

6. Hisham $\mathrm{R}, \mathrm{Ng}$ CJ, Liew SM, et al. Why is there variation in the practice of evidence-based medicine in primary care? A qualitative study. BMJ Open 2016;6:e010565.

7. Williams B, Perillo S, Brown T. What are the factors of organisational culture in health care settings that act as barriers to the implementation of evidence-based practice? A scoping review. Nurse Educ Today 2015;35:e34-e41.

8. Spoont M, Greer N, Su J, et al. VA Evidence-based Synthesis Program Reports: In. Rural vs Urban Ambulatory Health Care: A Systematic Review. Washington (DC): Department of Veterans Affairs (US), 2011.

9. Ajzen I. The theory of planned behavior. Organ Behav Hum Decis Process 1991;50:179-211.

10. Walker JL. The use of saturation in qualitative research. Can J Cardiovasc Nurs 2012;22:37-46.

11. Woolf SH, Grol R, Hutchinson A, et al. Clinical guidelines: potential benefits, limitations, and harms of clinical guidelines. BMJ 1999;318:527-30.

12. Barghouti F, Halaseh L, Said T, et al. Evidence-based medicine among Jordanian family physicians: awareness, attitude, and knowledge. Can Fam Physician 2009;55:e6-e13.
13. Khoja TA, Al-Ansary LA. Attitudes to evidence-based medicine of primary care physicians in Asir region, Saudi Arabia. East Mediterr Health J 2007;13:408-19.

14. Aljunid SM, Zwi AB. Differences in public and private health services in a rural district of Malaysia. Med $J$ Malaysia 1996;51:426-36.

15. Kalantri SP. Getting doctors to the villages: will compulsion work? Indian J Med Ethics 2007;4:152-3.

16. Agarwal R, Kalita J, Misra UK. Barriers to evidence based medicine practice in South Asia and possible solutions. Neurology Asia 2008;13:87-94

17. Dans AL, Dans LF. The need and means for evidence-based medicine in developing countries. Evid Based Med 2000;5:100-1.

18. Sadeghi-Bazargani H, Tabrizi JS, Azami-Aghdash S. Barriers to evidence-based medicine: a systematic review. J Eval Clin Pract 2014;20:793-802.

19. Sempowski IP. Effectiveness of financial incentives in exchange for rural and underserviced area return-of-service commitments: systematic review of the literature. Can J Rural Med 2004;9:82-8.

20. Rossenblatt RA, Chen FM, Lishner DM, et al. The future of family medicine and implications for rural primary care physician supply: final report 125. Seattle: University of Washington 2010.

21. Weeks WB, Wallace AE. Rural-urban differences in primary care physicians' practice patterns, characteristics, and incomes. J Rural Health 2008;24:161-70.

22. Increasing access to health workers in remote and rural areas through improved retention: global policy recommendations. In, p. 72 p, Switzerland: World Health Organization 2010.

23. Rabinowitz HK, Paynter NP. The role of the medical school in rural graduate medical education: pipeline or control valve? J Rural Health 2000;16:249-53.

24. Taylor J, Wilkinson D, Blue IA, et al. Evidence-based rural general practice: barriers and solutions in South Australia. Rural Remote Health 2002;2.

25. Parsons JE, Merlin TL, Taylor JE, et al. Evidence-based practice in rural and remote clinical practice: where is the evidence? Aust $J$ Rural Health 2003;11:242-8.

26. Dans AL, Dans LF. The need and means for evidence-based medicine in developing countries. ACP J Club 2000;133:A11-2.

27. Risahmawati SE, Nishi T, Widodo DW, et al. A comparative assessment of attitudes, knowledge and self-perceived barrier to the practice of evidence based medicine in Japan and Indonesia. Journal of Medicine and Medical Sciences 2012:3:016-29.

28. Kahveci R, Meads C. Is primary care evidence-based in Turkey? J Evid Based Med 2009;2:242-51. 